\title{
Morphological study of flesh fly stages, Sarcophaga africa (Wiedemann) (Diptera: Sarcophagidae)
}

\author{
Abbas M. Faraj \\ Dept. of Pharmacognosy \\ College of Pharmacy \\ Hawler Medical University
}

\author{
Nabeel. A. Mawlood \\ Dept. of Plant protection \\ College of Agriculture \\ Salahaddin University
}

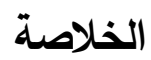

Sarcophaga africa تتضــن الدراســة الحالية وصـف تفصـيلي لأطوار ذبابة اللحم (Wiedemann)

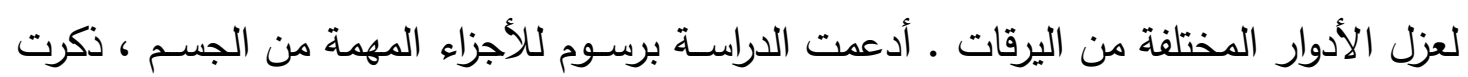

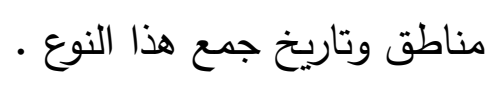

\begin{abstract}
This work includes a detailed morphological study of flesh fly stages, Sarcophaga africa (Wiedemann) which was wide spreaded in different localities of Kurdistan region-Iraq. The specimens were collected from March until July 2012. All stages have been described in detail. Taxonomic key have been formulated to isolate the larval instars. The important parts have been drawing. Some ecological information of the species, localities of collection and its date have been mentioned.
\end{abstract}

Keywords: Morphological study, flesh fly, Sarcophaga africa, Diptera, Sarcophagidae 


\section{Introduction}

Sarcophagidae (flesh flies) is a medium-sized family of Diptera with some 2600 known species worldwide (1). Most flesh flies are parasites or predators with relatively small number of species with true preference for vertebrate carrion (2).

The larvae of some species caused intestinal and traumatic myiasis in human $(4,5,6)$. Other species infect different kinds of domestic animals and cause cutaneous myiasis (7). Turki et al., (2006) reported a case of nasal myiasis of a 16-year-old girl in Turkey. The genus Sarcophaga in the broad sense contains more than 800 species (1). Many species develop in excrement, carrion and any kind of decomposing organic matter (9). Some species were predators on invertebrates (e.g. other insects, snails, earthworms, scorpions, crabs, etc.) $(10,11)$, and several species have been reported as being parasitic in vertebrates (mammals, turtles) (12). Some new Turkish records were given in the checklist and catalogue of Palaearctic and world species by (13) (1), respectively. Kara and Pape (14) listed 81 Turkish sarcophagid species with 43 new records. After that, Aslan (15) carried out a faunistic and systematic study and added another four species to the Turkish fauna.

Taxonomic revision of the genus was performed by $(16,4,17,18,1$ and 19). The aim of this study is to provide an update of the current knowledge description on all the stages of Sarcophaga africa and drawing the important parts.

\section{Materials and Methods}

\section{Collection of the adults}

The study depended on more than 20 specimens of each stage were collected in different places in Kurdistan region from March until July 2012 in different areas, these were:

Air net was used for capturing the flies on the flower or where seen hovering in the air near the flower and dead substance. After that, aspirator was used for the collection of the specimens inside the net to prevent any part of the flies from damaging or break down.

\section{Pinning and preserving of specimens}

The specimens were killed by freezing for $48 \mathrm{hr}$. after that pinned by using suitable volume insect pins (Labels will go on the pin below the specimen, all information which was about the species was recorded, the name of the collector, location and the date of collection), and preserving in the insect collecting box, treated with (Seven 85\%) and Naphthalene balls in order to keep the pest away. 


\section{Specimen examination (investigation)}

The specimen examined by binocular dissecting microscope, compound microscope and digital computerized microscope for studying the minute part of the body, after that these parts were drawn using ocular micrometer and the length of the parts were measured by using linear micrometer, and for identification of the genus and species were resorting for taxonomical key for the different authors.

\section{Collection of the immature stages}

The $1^{\text {st }}-3^{\text {rd }}$ instars larvae were collected in two ways:-

a. Direct collected from animal feces, carrion, decaying meat and decomposing organic matters.

b. Females were reared on decaying meat in cages until they larviposited through 5-3

days reached the third instar.

c. The pupa collected after about 12-15 days of larviposition from collection area.

\section{Dissection}

The morphology of the adult and immature were studied by using dissecting microscope, while the minute parts were studied by the preparation of microscope slide, by putting the live larvae in hot water just below boiling point for killing, the adult placed in a small beaker contained water warming it to boiling temperature for (4-5) minutes to soften their parts and to prevent it from breaking. To prepare the slides for microscopical examination, the larvae and adults can be dissected by using two fine pins. Then the required parts (The head and abdomen of all stages) were placed in small beaker containing $\mathrm{KOH} 10 \%$, placed on fire with shaking for about (4-5) minutes for dissolving fatty materials of the body and destroying the muscles. After that it was placed in distilled water for (2-3) minutes in order to neutralize the alkali. Mouth parts were placed in ethyl alcohol $50 \%$ and dissected under binocular microscope to obtain the different parts, so that the abdomen was placed in ethyl alcohol $50 \%$ and dissected under microscope to obtain the terminalia, these parts were transferred to ethyl alcohol $75 \%$ and $100 \%$, respectively for 2 minutes/concentration to dehydration of water, so that these parts were placed in xylol for two minutes, finally they were mounted by canada balsam to prepare slides for microscopical examination. 


\section{Results}

\section{First: Taxonomic Key for isolated larval instars}

1. The larva without anterior spiracles ........................First instar.

- The larva with anterior spiracles (Fig. 1d, 2b) ......................2

2- Posterior spiracles with two slits (Fig. 1e) ................ Second instar.

- Posterior spiracles with three slits (Fig. 2d) ...............Third instar.

\section{Second: Description}

\section{A. Description of immature}

1. First larval instar

Length 2.6-4.1 mm; width 0.5-0.7 mm, body smooth, first segment subtruncate shaped in front, anterior spinuous areas of dorsal surface present on segments 2-8; spines with 1-3 teeth posterior spinuous areas of ventral surface appear on the segments 6-12, cephalopharyngeal skeleton (Fig.1a) well developed; mouth hook moderately decurved and without serration, parastomal sclerite indistinct, dorsal arch very slender, hypostomal sclerite narrow, dorsal cornua moderately sclerotized and gradually tapering posteriorly, ventral cornua moderately sclerotized and slightly shorter than the dorsal cornua, anterior spiracles absent, posterior spiracular hidden in moderately deep pit; posterior papillae small and conical in shape. Anterior spiracles were wanting; each posterior spiracles (Fig.1b) consist of two slits, oval in shaped and combined together at the base and without the peritreme.

\section{Second larval instar}

Length $7.7-9.2 \mathrm{~mm}$; width $1.3-2 \mathrm{~mm}$, all segments of dorsal surface covered with spines, lateral pad of segment 5 covered with spines at anteroventral area of segment 5 divided by transverse spineless space on either side of medial line, ventral area of segment 4 between spine bands with a few microtubercles, ventral area of segments 5-11 covered with spines, Cephalopharyngeal skeleton (Fig.1c,d) well developed, hook part of mouth hook moderately curved and equaled in length to basal piece, dental sclerite small and subtriangular in shape, labial sclerite narrow and feebly scleretized, hypostomal sclerite $\mathrm{H}$-shaped, parastomal sclerite slender, rod-like and nearly straight. dorsal cornua divided, its upper part slightly shorter than the lower part, the window between these was narrow, ventral cornua undivided and distinctly shorter than the dorsal cornua, posterior spiracular pit deeper than the first instar and encircled with six pairs of small papillae spineless, anterior spiracles (Fig.Id) with 14 small papillae, papillae fan shaped without imagination, posterior spiracles (Fig.1e) sub oval-shaped, with two slits, the distance between these 
spiracles approximately half diameter of a single spiracle, outer slits slightly curved and longer than inner one.

\section{Third larval instar}

Length 10.0-15.9 mm, width 2.0-3.4 mm, similar in form with second instar with some exceptions, ventral areas of segments 4-11 between spine bands completely covered with spines, cephalopharyngeal skeleton (Fig. 2a) well developed, hook part of mouth hook strongly curved and slightly longer than the basal piece, dental sclerite visible, subtriangular in shape, labial sclerite bread and moderately scleretized, posterodorsal process prominent, sub-hypostomal sclerite large and oval shaped, parastomal sclerite rod-like and slightly encurved upper ward, dorsal cornua distinctly divided and upper parts slightly shorter than the lower one, the window between these was large, ventral cornua bread, undivided and shorter than the dorsal surface, sinus between dorsal and ventral surface was big . Anterior spiracles (Fig. 2b) with 14 papillae, papillae in a single row, fan shaped, without imagination, posterior spiracular pit deeper than the second instar, with six pairs of fleshly papillae without spines except a few spines on papillae of lower part, distance between inner papillae and outer together, and the distance between the inner and median papillae was nearly equal to the distance between the median and outer papillae, (Fig. 2c). posterior spiracles (Fig. 2d) oval shaped, with three slits, the distance between the spiracles approximately to twice the time of the width of a single slits, medium slit was straight and longer than the outers, peritreme thickness and in complete ventro-mesially, the distance between its tails was narrow, button present, feebly visible and circulated shape, located between the tails of peritreme and the basis was of the slits.

\section{Puparium}

Length 7.1-9.0 mm, barrel shaped; reddish to brown in color. The anterior spiracles of the larva appear as minute processes situated anteriorly, pupal horns absent, posterior spiracles with small flange ventromedially on the peritreme.

\section{B. Description of the adults}

Body: Longitudinal oval shaped, length 10-13 mm.

Male: Head (Fig.1a) Black; Inner vertical bristles week developed and slightly curved; post vertical bristles long and well-developed; fronts and Para frontal brown with golden pollen; frontal stripe brown with golden pollen each sides with 11-12 crossed bristles; Gena brown with golden pollen, compound eyes semi-holoptic, red brown, oval shaped occupy about third-fifth of the anterior region of the head, ocular triangle 
setae with a pair of ocular bristles. Antenna (Fig. 3a) aristate form with three segments, dark brown, 1.0-1.2 mm long, first segment cup shaped with numerous of short setae, second segment cup shaped longer twice and half time than first ones, third segment oval shaped longer twice and quarter times as long as the second segment. Arista little longer than the third segment and pulomose to the 2/ 3 of apical distance. Maxillary palp clavate shaped, dark brown with 9-11bristles. Labrum-epipharynx (Fig. 3b) cone shaped, brown, apodem black, rod shaped, apical cup shaped, epipharynx (Fig. 3b) tubular shaped. Female: Head similar to the male but outer vertical bristles was present; 1 pair of reclinate and 2 pairs of proclinate fronto-oribital bristle; Compound eyes smaller; frontal stripe wide.

Thorax: Grayish with distinct black longitudinal stripes and black setae; chaetotaxy :- acrostichal bristles $4+0$; dorsocentral bristles $0+3$; notopleural bristles 4; humeral bristles 3; post-humeral bristles 2; intra- alar bristles $0+2$; supra- alar bristles 2; presuture bristle 1; post- alar bristles 2; scutellum bristles $4+1$; propleural bristle 1; stigmatal bristle 1 ; sternopleural bristles $2: 1$; pleuron grey with dark slivery pollen; mesothoracic spiracels oval shaped, yellow, $0.56-0.49$ $\mathrm{mm}$ length; prosternum grey, with slivery pollen and long, black setae; mesopleuron grey, with dark slivery pollen, its lower margin with 6 long bristles; hypopleuron with dark slivery pollen, its lower margin with a row (9-8) long bristles, metathoracic spiracels yellow, circular shaped. Wings hyaline, Tegula black; basicosta orange; vein with a row of; $\mathrm{R} 4+5$ vein setulose in proximal 0.5 or less of distance to $\mathrm{r}-\mathrm{m}$; thoracic squma retriangul were shaped with fine yellow pubescent; upper squama similar to the thoracic squama but smaller. Legs dark brown-black, femur in fore legs (Fig.3c) with a pair of rows of long bristles on the antero and postero dorsal surface; fore tibia with 3 bristles on antero-dorsal surface, 1 bristle on the posterodorsal surface; mid tibia with 1 bristle on each anterodorsal and postero dorsal surface; hind femur with a row of bristles on the anterodorsal surface, and non complete row on anteroventral surface; hind tibia with arrow of bristles on antero-dorsal surface and 5-4 bristles on postero-dorsal surface.

Abdomen : Black and slivery gray chekered pattern; posterior angle of each tergits with 3-2 bristles; The sternites black, posterior margin of the sternites 5-2 with a row of bristles; middle margin of fifth abdominal sternite deeply emarginated (Fig. 3d). Abdomen in females is similar to 
those in males but differed by the tergites without black spots and fifth sternite oval shaped.

Male terminalia: Tergite 6 reduced; sternite 6 nearly plate shaped, left arm reach to the syntergosternite $7+8$, right arm and failing to reach to the syntergosternite $7+8$; syntergosternite $7+8$ (Fig. 3e) cup shaped with moderate dense of short hairs, posterior margin with four long bristles; Tergite 9 cup shaped, arms strongly curved, the surface covered with high dense of hairs; Paralobs (Fig. 3f) semi-parallel, one-third of basal black with dense, long bristles; Anal cerci (Fig. 3g) hook shaped; Phalloapodeme cylindrical; Pregonite black, hook shaped, outer margin with 4-3 bristles. Postgonite hook shaped, outer margin with 3-2 bristles; Phallus (Fig. 3h) dark brown-black, basiphallus oval without epiphallus, paraphallus clavate shaped, hypophallus nearly ring shaped, acrophallus very short, cylindrical shaped; Ejaculatory yellow,apical part dark yellow; length $0.45-0.450 \mathrm{~mm}$.

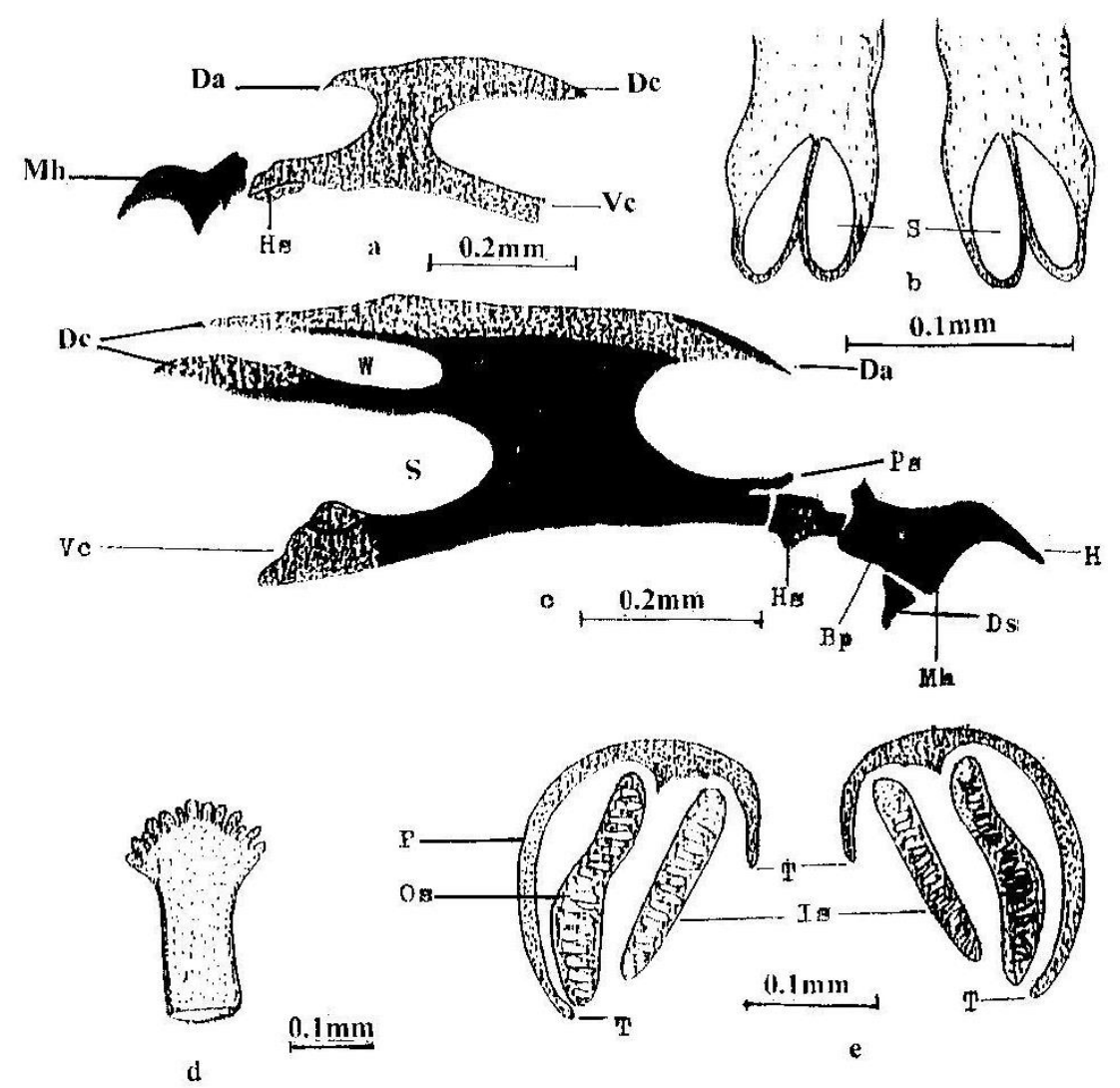

Fig. 1 Sarcophaga africa (Wiedemann)

$\mathrm{a}, \mathrm{b}=$ First instar; $\mathrm{c}-\mathrm{e}=$ Second instar.

a- Cephalopharyngeal skeleton $b$. Posterior spiracles

c. Cephalopharyngeal skeleton (lateral view) d. Anterior spiracle

e. Posterior spiracles

$\mathrm{Dc}=$ Dorsal cornua; $\mathrm{Ds}=$ Dental sclerite $\mathrm{H}=\mathrm{Hook} ; \mathrm{Hs}=$ Hypostomal sclerite;

Is= Inner slite;

Ls= Label sclerite; $\mathrm{Mh}=$ Mouth hook; Os= Outer slit; $\mathrm{P}=$ Peritreme;

$\mathrm{Ps}=$ Parastomal sclerite; $\mathrm{T}=$ Tail $; \mathrm{Vc}=$ Ventral cornua; $\mathrm{W}=$ Window. 


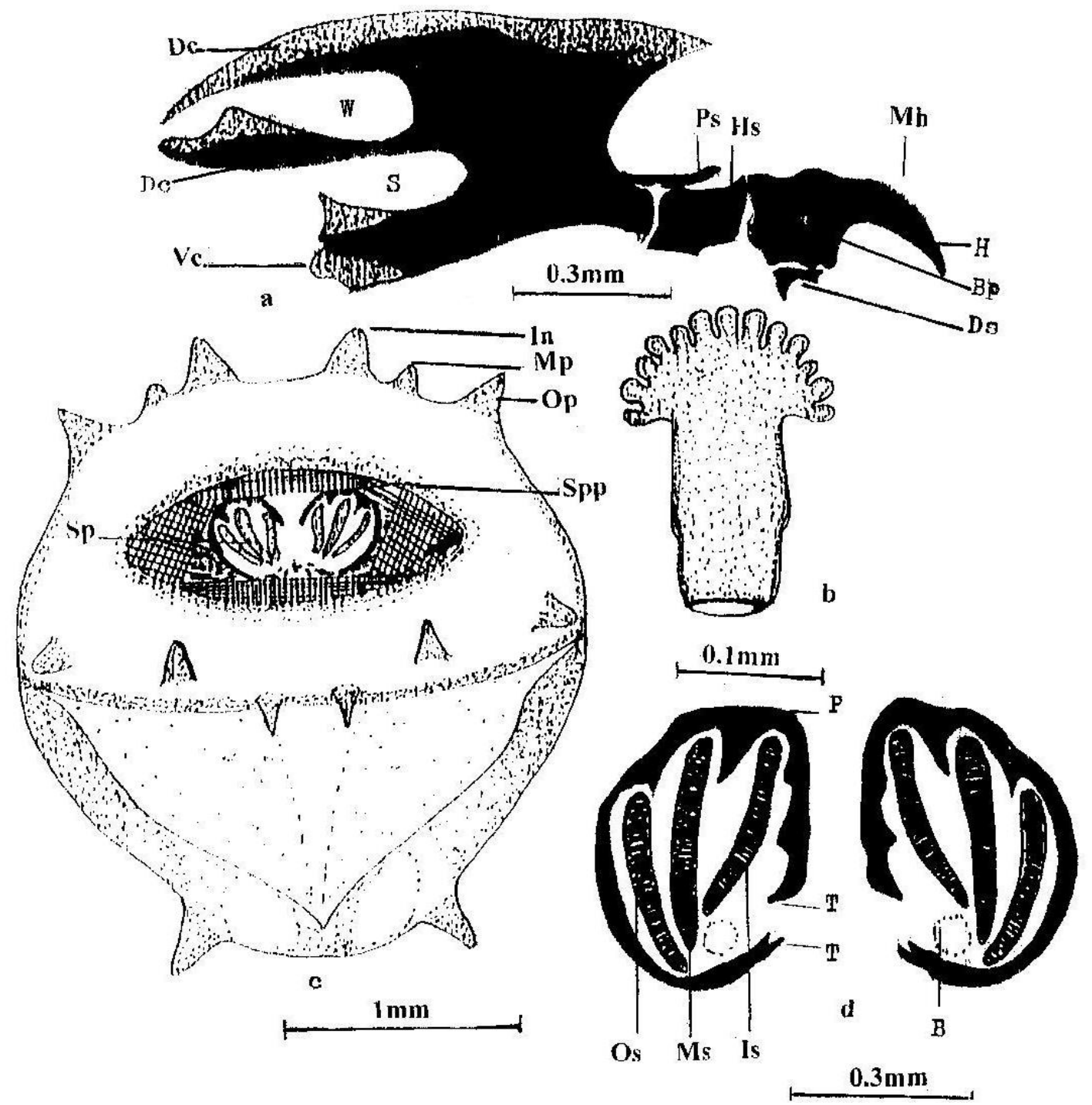

Fig. 2 Sarcophaga africa (Wiedemann), Third instar

a. Cephalopharyngeal skeleton (Lateral view); b. Anterior spiracle; c. Anal view of $12^{\text {th }}$ segment; d. Posterior spiracles.

$\mathrm{B}=\mathrm{Button} ; \mathrm{Bp}=\mathrm{Basal}$ piece; $\mathrm{Ds}=$ Dental sclerite; $\mathrm{H}=\mathrm{Hook}$ Hs= Hypostomal seclerite; Ip= Inner; papillae; Is= Inner slit; Mh= Mouth hook; Mp= Medium papillae; $\mathrm{Ms}=$ Medium slit; $\mathrm{Op}=$ Outer papillae; $\mathrm{Os}=$ Outer slit; $\mathrm{P}=$ Peritreme; $\mathrm{Pdp}=$ Posterior dorsal process; $\mathrm{Ps}=$ Parastomal sclerite; $\mathrm{S}=$ Sinus; $\mathrm{Sp}=$ Spiracle; $\mathrm{Spp}=$ Spiracular pit $\mathrm{T}=$ Tail $; \mathrm{vc}=$ Ventral cornua; $\mathrm{W}=$ Window . 

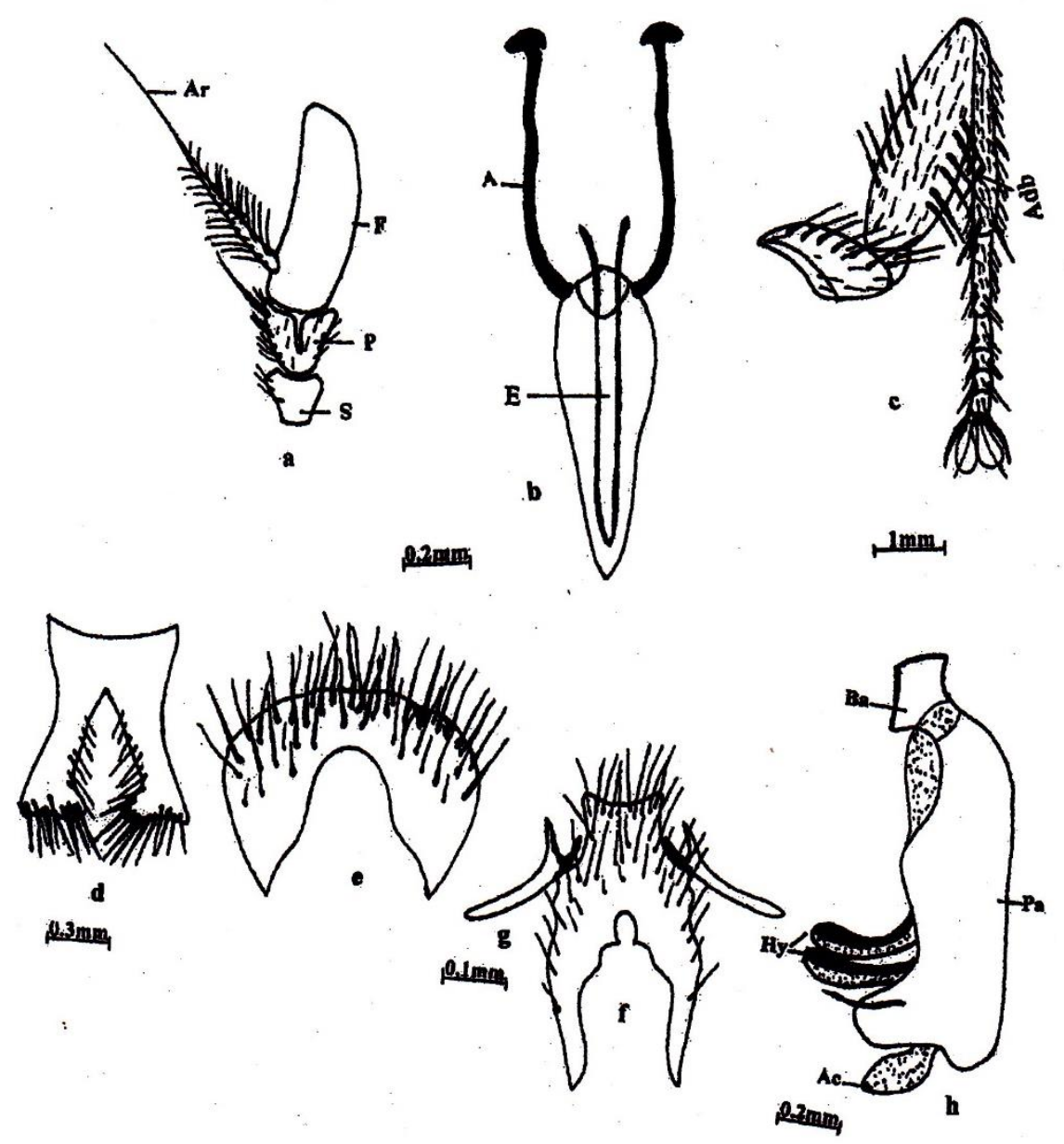

Fig. 3 Sarcophaga africa (Wiedemann)

a. Antenna b. Labrum-epipharynx

c. Fore leg d. $5^{\text {th }}$ abdominal sternite

e. Syntergosternite $7+8$ f. Paralobs

g. Anal cerci h. Male genitalia

A= Apodem; Ac= Acrophallus; Adp= Antero-dorsal bristle; $\quad$ Ar $=$ Arista;

Ba= Basiphallus; E= Epipharynx; F= Flagellum $\mathrm{Hy}=$ Hypopallus;

$\mathbf{P}=$ Pedicel; $\quad$ Pa= Paraphallus; $\quad \mathrm{S}=$ Scabe. 


\section{References}

Aslan, A. 2006. Studies Related with Sarcophagidae (Diptera) Fauna in Eskiflehir. Osman Gazi University, Master of Science Thesis, Department of Biology, (in Turkwash). 66 pp.

Chigusa, Y.; Tanka . K .; Mastuda, Y .; Saakai, Y .; Ikadtsu, Y . and Baba, K. 1994. Two cases of otomyiasis caused by Sarcephaga peregrine and $S$. similwas . (Diptera : Sarcephagidae) . jap. j. Sanit . Zoll., 45 : 7pp.

Colwell, D.D. and O'Connor, M. 2000. Scanning electron microscopy of sarcophagid (Diptera) larvae recovered from a case of human cutaneous myiasis. J. Med. Entomol., 37: 5pp.

Dahlem, G.A. 1991. Sarcophagidae (Oestroidea). In: Stehr, F.W. (Ed.), Immature Insects, vol. 2. Kendall/Hunt Publishing Company, Dubuque, IA, pp. 871-873. 975pp.

Ebejer, M.J. 2000. Description of third instar larva and puparium of Blaesoxipha calliste Pape (Diptera: Sarcophagidae). Studia dipterologica. 7: 121-124.

Frakas, R.; Hall, M. J. and Kelemen, F. 1997. Wound myiasis Sheep in Hungary. Veter. Parasit ., 69 : 133-144.

Kara, K. and Pape, T. 2002. Check list of Turkish Sarcophagidae (Insecta, Diptera) with new records. Mitteilungen aus dem Museum fur Naturakunde in Berlin-Deutsch Entomologische Zeitschrift. 49: 291-295.

Lopes, H. S. 1969. Family Sarcophagidae. In N Papavero (ed.), A catalogue of the Diptera of the Americas south of the United States 103: 1-88. Departamento de Zoologia, Secretaria da Agricultura, São Paulo.

Me'ndez, J. and Pape, T. 2002. Biology and immature stages of Peckia gulo (Fabricius, 1805) (Diptera: Sarcophagidae). Studia Dipterologica 9, 371374.

Merdivenci, A. 1966. The systematics of the parasites of Turkey. Istanbul University Science Faculty S.B. 31: 73-108.

Pape, T. 1987. The Sarcophagidae (Diptera) of Fennoscandia and Denmark. Fauna Entomologica Scandinavica, 19, 1-203 (2 pl. Leiden and Copenhagen).

Pape, T. 1996. Catalogue of the Sarcophagidae of the world (Insecta: Diptera). Memoirs of Entomology International 8,1-558.

Povolný D, Verves Y. 1997. The flesh-flies of Central Europe. Munchen: Spixiana, supplement 24.

Richet, R.; Blackith, R.M. and Pape, T. 2011. Sarcophaga of France (Diptera: Sarcophagidae) Sofia, Bulgaria: Pensoft Publishers; 2011. pp. 1-327.

Rohdendorf, B. B. 1937. Fam. Sarcophagidae (Part 1). Fauna of the USSR, New Series, No. 12, Moscow \& Leningrad (In Russian and German).

Smith, K.G.V. 1986. A manual of forensic entomology. The Trustees of the British Museum. Natural History), London.

Turki, M; Afsar, I.; Özbel, Y.; Şener, A. G.; Üner, A. and Turker, M.(2006) A Case of Nasomyiasis Whose Agent Was Sarcophaga sp., Acta Parasitologica Turcica, 30 (4): 330-332. 
Valle de Sales, K.; Hidalgo-Friciello, R.; Marinete-Amorim, T. and SallesGaze^ta, G. 2004. Relato de larva de sarcophagidae (Diptera) parasitando Cebus apella (L., 1758) (Primate: Cebidae) no zoológico de sorocaba, estado de Sa ̃o paulo, Brasil. Entomol. Vect., 11 (2), 317-321.

Verves, Yu.G. 1986. Family Sarcophagidae. In: Soos A. and Papp L.(eds.), Catalogue of Palaearctic Diptera, 12. Akademiai Kiado, Budapest.

Zumpt, F. 1965. Myiasis in man and animals of the Old World . A textbook for Physians. Veterinarians and Zoologists London. Iv +267 pp. 